\title{
TOWARDS THE DEVELOPMENT OF A BRIDGE INSPECTING AUTOMATED DEVICE
}

\author{
Anna $\mathrm{McCrea}^{\mathrm{a}}$ and Denis A.Chamberlain ${ }^{\mathrm{b}}$ \\ ${ }^{a}$ Senior Lecturer, School of Construction, Economics and Management, South Bank \\ University, London. \\ ${ }^{b}$ Head of the Construction Robotics Unit, Department of Civil Engineering, City University, \\ London.
}

\begin{abstract}
There are, as yet, no commercially viable robotic or automation solutions in the field of reinforced concrete bridge inspection, these structures suffering from deterioration of both their concrete and steel reinforcement elements.

In the manual inspection activity for defect diagnosis and repair specification, visual assessment is supplemented by a range of non-destructive tests. There are a range of such tests, each directed at a particular aspect of the decay problem. For automation to be effectively developed, the operational requirements of each test probe needs to be defined in relation to many combinations of access and location geometry. A library of these 'benchmarks' is in fact needed for use in the research and development of useful automation devices. They can be used in computer based simulation studies to provide work-pieces which test the capability of concept devices and aid their evolution. This is important for the development of both the access vehicle (ROV) and the robot mounted on it. The need for this rational approach is clear in that existing ROV's would fair badly when attempting even the simplest 'benchmark' task. The application and value of the 'benchmark' approach to the automation problem has been demonstrated in the consideration of two device concepts for bridge inspection.
\end{abstract}

KEY WORDS: Concrete decay, inspection methods, benchmarks, dextrous envelope, inspection facility.

\section{INTRODUCTION}

The application of automation and robotics in the fields of monitoring and inspection, as a means of achieving greater reliability, productivity and safety, is gathering interest [1]. Such a role has been clearly established in respect to bridge inspection. 
However, the emergence of a commercially successful device depends on many technological and human factors. This paper seeks to address some of these, particularly those which will influence research and development strategy.

Experience related from various sources indicates that the inadequacy of the current manual approach to bridge survey is most acute where access is particularly difficult, extensive areas are to be tested or non-standard inspection techniques are required. It relation to access, there are in the UK some 300 important bridges for which this poses extreme difficulties. These are typically tall viaducts, multi-span structures and large bridges over water. The reguirements for the remaining 100,000 or so bridges would without substantial progress in cost-effectiveness, be best met by current methods. In the research, a range of bridge and component geometries have been gathered for the development of a 'benchmark' library. The requirements and activity for inspection have been built into these so that prospective automation devices can be evaluated on a correct basis.

After the content and role of 'benchmarks' are introduced, their use is discussed in the context of the simulation and evaluation of inspection automation concepts using the GRASP computer software. In conclusion we see that the 'benchmark' approach to device development is realistic, technically sound and essential.

\section{DETERIORATION OF BRIDGES}

There are two main and closely associated, forms of decay in bridges $[2,3]$, deterioration of concrete and corrosion of reinforcing steel. Several factors contribute to the occurrence of these phenomena. Where no specials requirements are set in the testing for such decay, information is required on the (i) visual appearance of the surface including the and location of cracks and rust stains, (ii) likelihood of reinforcement corrosion, (iii) delamination, (iv) cover to the reinforcing bars, (v) depth of the carbonation front and (vi) level of chloride contamination.

\section{INSPECTION METHODS AND AUTOMATION FACTORS}

Of all the available non-destructive tests, only a few are clearly amenable to automation. The factors influencing this are the weight, size and robustness of the probe, method of handling, vibration aspects, reliability, accuracy, data size and interpretative methods [4]. Some useful analysis of these attributes is given elsewhere [5].

Supportive sensing would be required for each of the above. This is necessary for collision avoidance, contact force limitation, positioning and orientation of probes relative to the inspected surface. A distributed processing system combined with A/D conversion capability would be needed to handle the support sensing.

The probe operational characteristics are vital considerations in the correct definition of a robot or automation device. Size and weight of the probe together with the presentation to surface requirements will inevitably influence the actuator and dextrous envelop specification. Here the 'dextrous working envelop' is quite distinct from the 'positional working envelop' commonly used in suppliers descriptions of robots.It accounts for the orientation and positional requirements and is invariably less extensive. 
Determination of the dextrous envelop can be achieved explicitly using inverse kinematics or somewhat easier by experimentation with a robot simulation facility. A combination of these approaches have been employed with the aid of simulations built using the GRASP computer software [6].

\section{INSPECTION TASK BENCHMARKS}

In order to establish a sound basis for task led research and development of inspection automation devices, it is necessary to focus on clearly defined task requirements or 'benchmarks'.

Here a 'benchmark' is taken to comprise geometric descriptions of the zone requiring inspection and permissible access to it, specification of the probes to be handled and the point, line and area coverage requirements. An example is illustrated in Fig. 1, where a robot located in region $A$ is required to detect the steel reinforcement over area B. Impulse hammer readings are also required at positions a-f on $\mathrm{B}$.

Clearly an extensive library of 'benchmarks' is necessary, each translating into the dextrous requirements for the inspection robot. In this work, both 'benchmarks' and robots has been modelled using the robot simulation software.

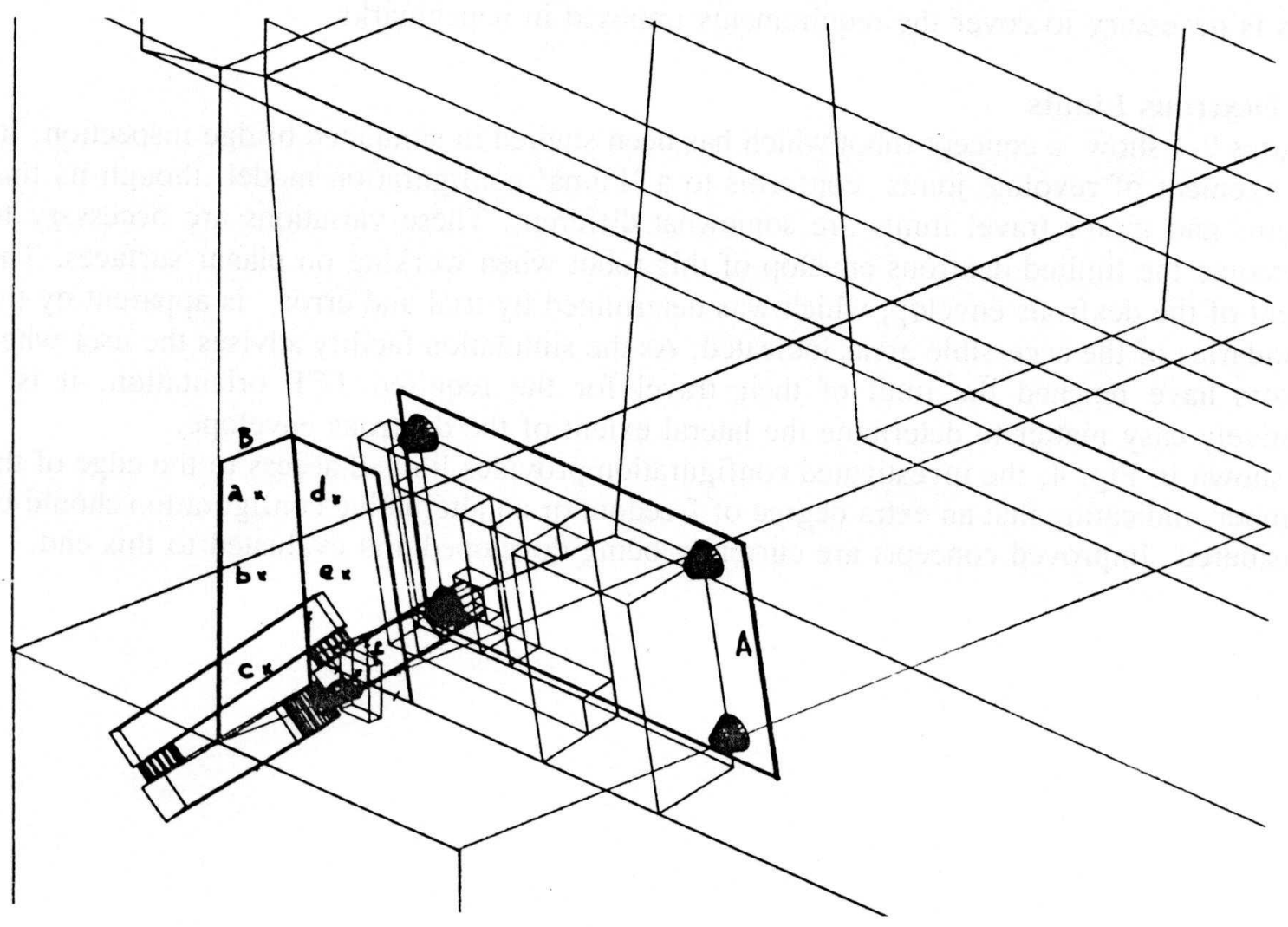

Figure 1. A Benchmark Example 


\section{SIMULATION STUDIES}

\subsection{Computer Modelling}

The simulation software GRASP comprises a solid modeller with full boolian operations and a process modeller supporting both standard and user defined robot joint configurations. It can be used interactively or by running programmes prepared in a descriptive high level language. It supports 'tracks' which can be used to define a task in terms of motion paths between set points. The object orientated basis of the facility makes these a useful attribute for benchmark development. On executing a track based programme the cycle time and collision risks are determined, the former clearly dependent on the maximum speed and accelerations prescribed for the robot motors.

In simulation studies robots divide into two classes, those matching available industrial robots configurations and non-standard robots for which the user must supply the inverse kinematic relationships.

Robots with more than 6 DOF's can be simulated by setting configuration rules. Once the combination of revolute and prismatic joints are set, the architecture or flesh is added. This is not a cosmetic exercise as collision may depend on it.

The robot tools, its inspection probes, are readily interchangeable in the simulation, these mounted on the robot's TAP (Tool Attachment Point). Each tool, however, has its own TCP (Tool Centre Point) which is required to follow track paths. Depending on the type of probe, this corresponds to its contact point or centre of contacting area with the inspected surface. Probes are also assigned contact axes which force them to align with track owned targets. This is necessary to cover the requirements imposed in benchmarks.

\subsection{Dextrous Limits}

Figures 2-4 show a concept robot which has been studied in simulated bridge inspection. Its arrangement of revolute joints conforms to a 'Puma' configuration model, though its link lengths and motor travel limits are somewhat different. These variations are necessary to overcome the limited dextrous envelop of this robot when working on planar surfaces. The extent of the dextrous envelop, which was determined by trial and error, is apparent by the boundaries of the accessible areas indicated. As the simulation facility advises the user when motors have reached the limit of their travel for the required TCP orientation, it is a relatively easy matter to determine the lateral extent of the dextrous envelope.

As shown in Fig. 4, the investigated configuration provides limited access to the edge of the member, indicating that an extra degree of freedom or an alternative configuration should be considered. Improved concepts are currently being developed and evaluated to this end. 


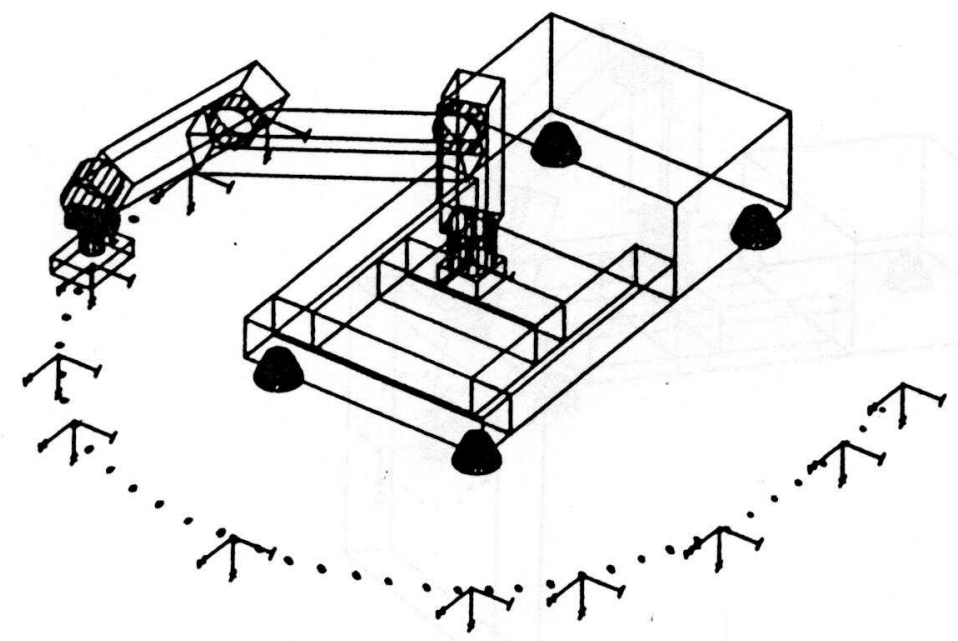

Fig.2 Dextrous Limits To Plan

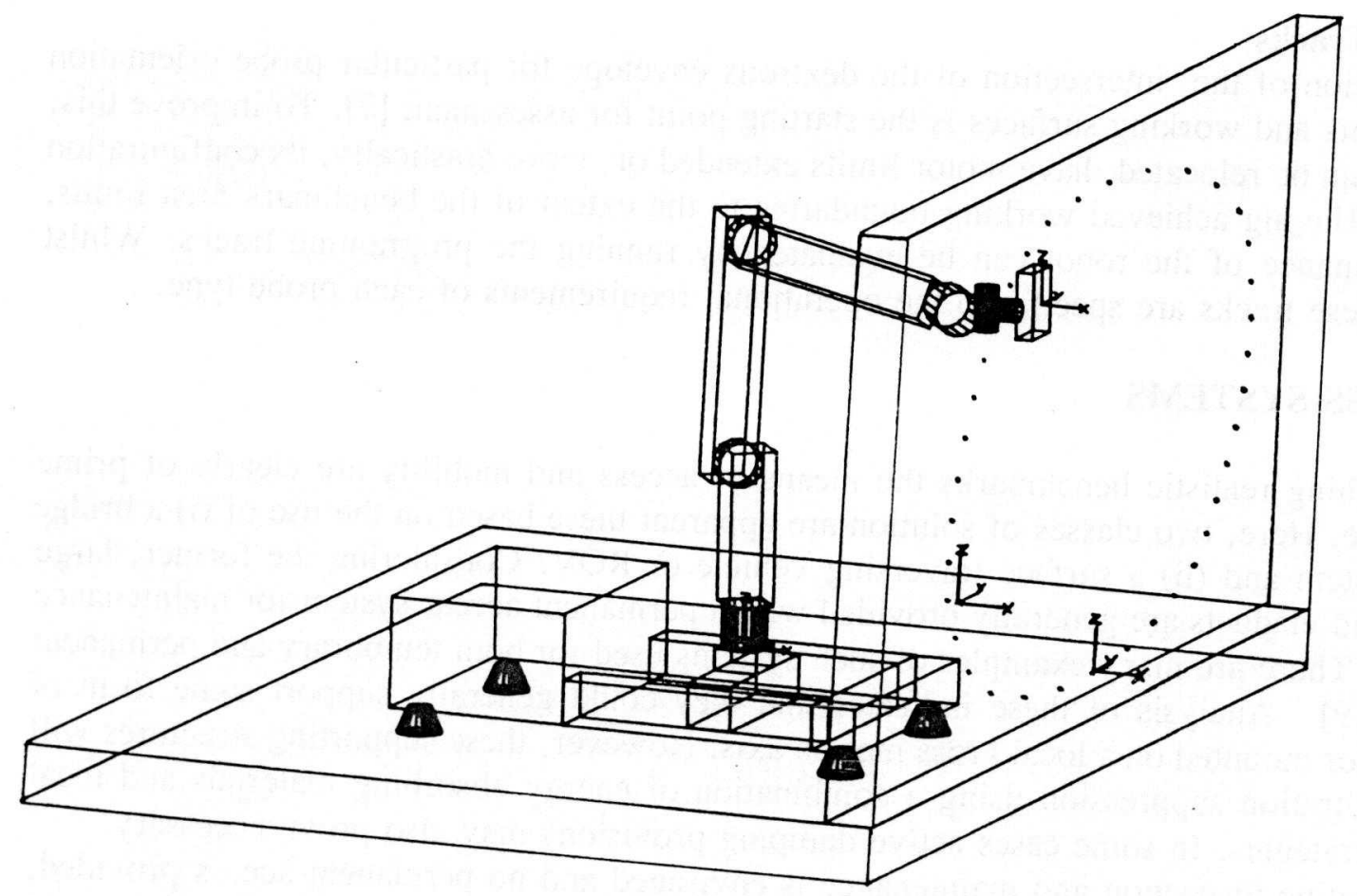

Fig.3 Dextrous Limits To Upstand 


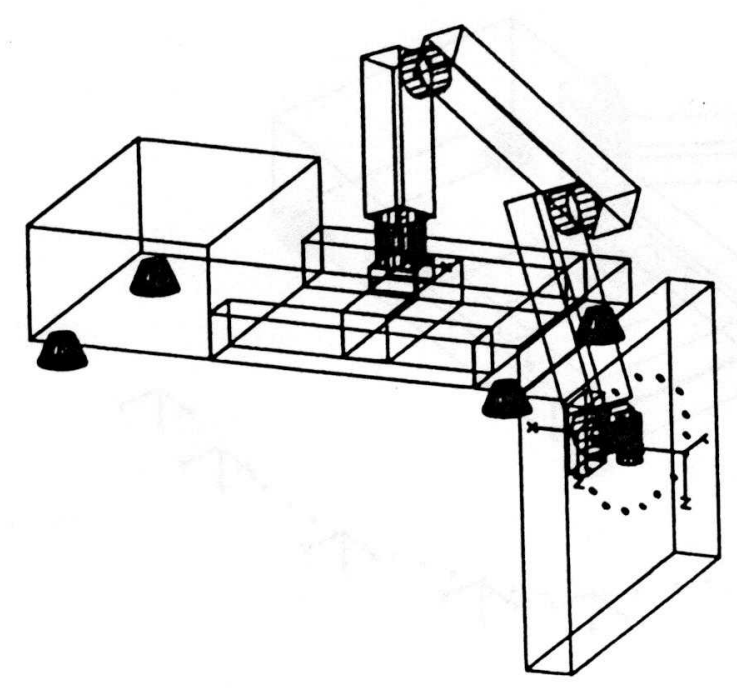

Fig. 4 Dextrous Limits To Overhang

\subsection{Task Tracks}

Determination of the intersection of the dextrous envelope for particular probe orientation requirements and working surfaces is the starting point for assessment [7]. To improve this, the robot can be relocated, have motor limits extended or, more drastically, its configuration modified. Having achieved working boundaries to the extent of the benchmark area limits, the performance of the robot can be evaluated by running the programme tracks. Whilst similar, these tracks are specific to the operational requirements of each probe type.

\section{ACCESS SYSTEMS}

In establishing realistic benchmarks the means of access and mobility are clearly of prime importance. Here, two classes of solution are apparent these based on the use of (i) a bridge access system and (ii) a surface traversing vehicle or ROV. Considering the former, large bridges and viaducts are generally provided with a permanent access system for maintenance purposes. There are many examples of such systems used for both temporary and permanent access $[8,9]$. Analysis of these indicates that they could generally support some form of manipulator mounted on a local gross motion axis. However, these supporting structures will require vibration suppression using a combination of energy absorbing materials and local bracing strategies. In some cases active damping provisions may also prove necessary. Where routine inspection and maintenance is envisaged and no permanent access provided, demountable tracking could be employed, this attached by permanent fixing points and brackets. Such provisions should automatically be considered in the design of new structures. Two concepts based on these ideas are now considered. 


\subsection{System 1}

Figure 5 shows an inspection robot suspended from two trolleys which run on tracks to each side of the deck. This arrangement enables the device to inspect the sides and under-side. A separate arrangement of clamps or tracks along the piers with vertical trolleys would enable pier inspection to be undertaken. The motion of the trolleys and the robot would be provided by an arrangement of vacuum grippers and long stroking pneumatic actuators for which a supply line could be supported on the trolleys.

\subsection{System 2}

With this system the robot platform is suspended form clamps attached at junctions between the under-side of the deck and the tops of piers. The powered platform would ascend and descend along the piers with the aid of two powered cable winches located at the ends. Where the under-side of the deck requires inspection, the same platform would move by means of the cable winches, these clamped to the structure at the ends of each inter-pier span. This system is similar to one proposed for building inspection [10].

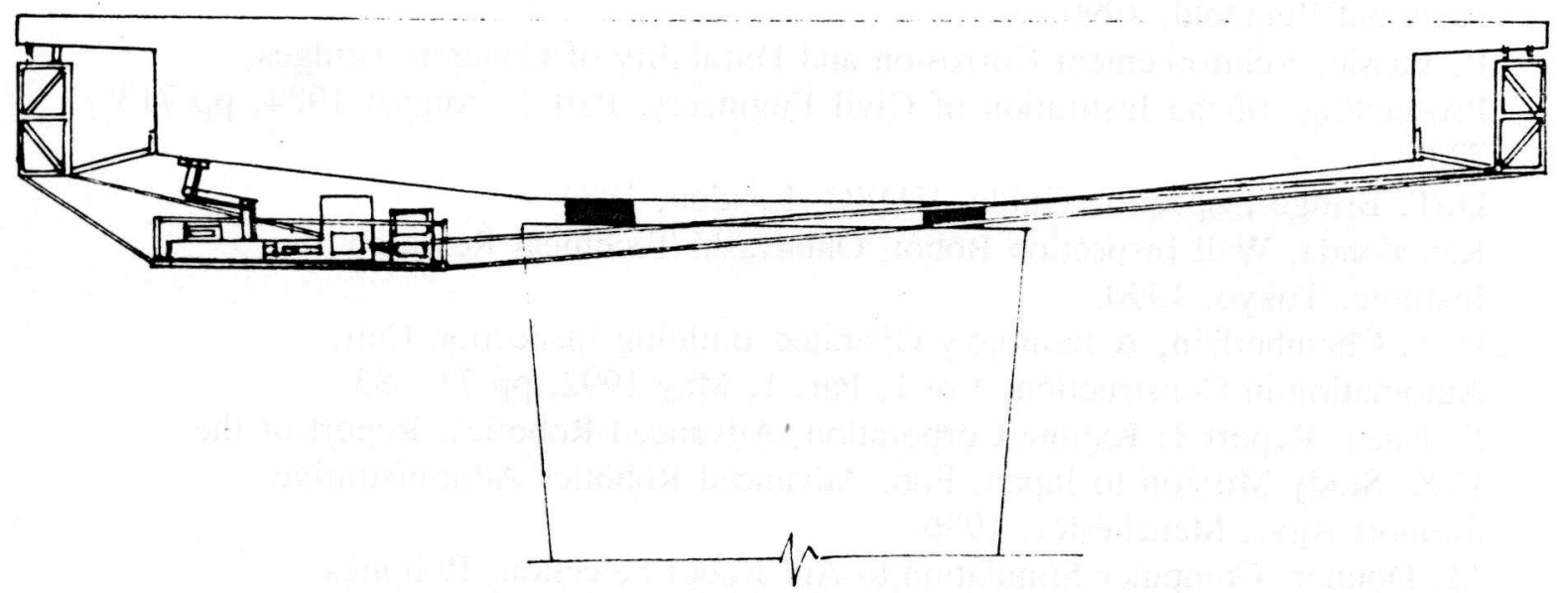

Figure 5. System 1

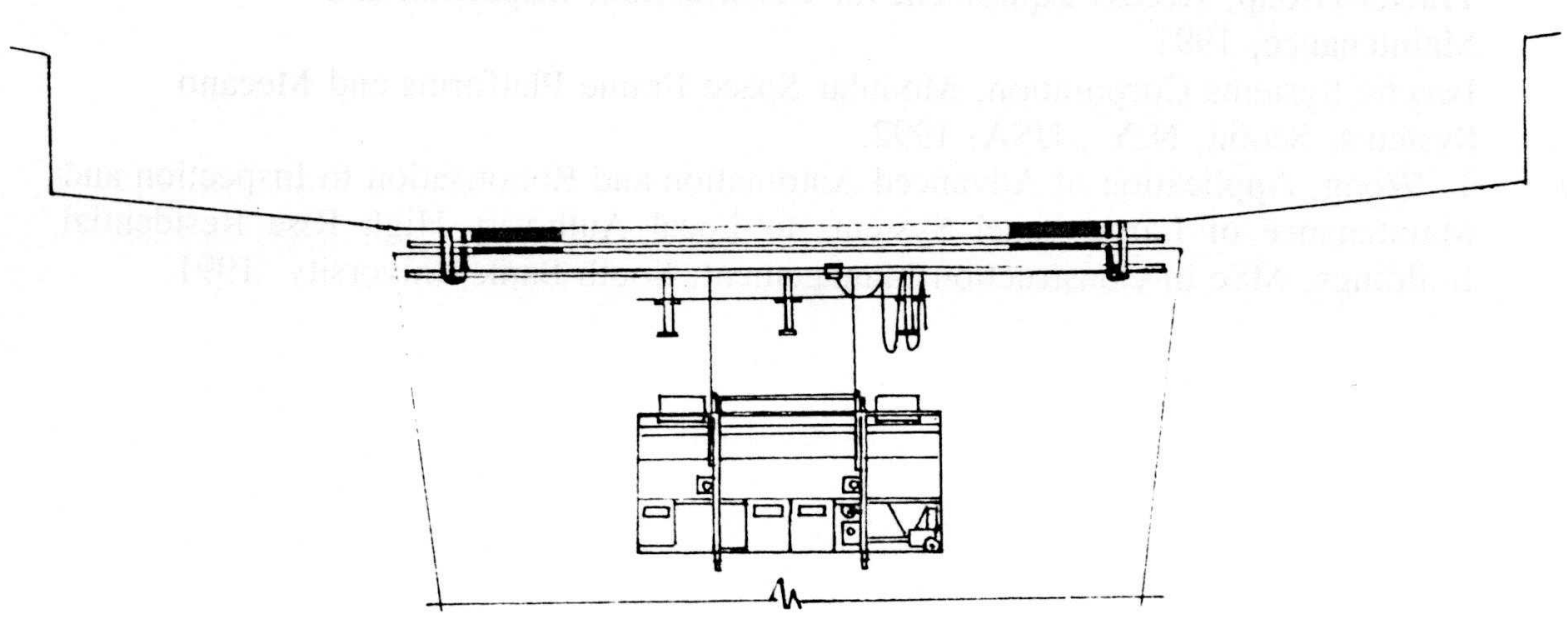

Figure 6. System 2 


\section{CONCLUSIONS}

The background to the robotic inspection of bridges has been presented. In the research and development of effective devices it is important that some sound basis for uniform assessment be determined. To this end the 'benchmark' concept has been introduced, this providing a precise means for evaluating and comparing robot concepts. These 'benchmarks' must cover the use of the major inspection probes, the purpose of which has been set out in summary form. The development of benchmarks and their use in robot evaluation have proved worthwhile. However, this work now needs extending to cover the full range of inspection methods with realistic target geometry. Costing information is currently being analyzed with a view to verifying a method for economic evaluation.

\section{REFERENCES}

1 S.M. Levy, Japanese Construction: an American Perspective, Van Nostrand Reinhold, 1990.

2 P. Vassie, Reinforcement Corrosion and Durability of Concrete Bridges, Proceedings of the Institution of Civil Engineers, Part 1, August 1984, pp 713 723.

3 DoT, Bridge Inspection Guide, HMSO, London, 1984.

4 Kabedohda, Wall Inspection Robot, Ohbayashi Technical Research Institute, Tokyo, 1990.

5 D.A. Chamberlain, A Remotely Operated Building Inspection Cell, Automation in Construction, Vol 1, Part 1, May 1992, pp 71 - 83.

6 F. Nazy, Report 1: Kajima Corporation, Advanced Robotics, Report of the U.K. Study Mission to Japan, Pub. Advanced Robotics Administrative Support Agy., Manchester, 1986.

7 M. Donner, Computer Simulation to Aid Robot Selection, Robotics Technology, edited by A. Pugh, Control Engineering Series 23, IEE, London, 1983.

8 Tractel Group, Access Equipment for Construction, Inspection and Maintenance, 1991.

9 Beeche Systems Corporation, Modular Space Frame Platforms and Mecano Systems, Scotia, N.Y., USA, 1992.

10 T. Wong, Application of Advanced Automation and Robotisation to Inspection and Maintenance of Large Panel Systems to Local Authority High Rise Residential Buildings, MSc in Construction Management, South Bank University, 1991. 